\title{
AULA.EDU: Un programa social de educación media superior a distancia en espacios alternativos no educativos
}

\section{AULA.EDU a social program of higher average distance education in alternative non- educational spaces}

\author{
Esthela del Socorro Medina-Tamez ${ }^{1}$ \\ Universidad Autónoma de Nuevo León \\ Dirección de Estudios de Nivel Medio Superior \\ Monterrey, México \\ esthela.medinatm@uanl.edu.mx
}

Recibido: 29 agosto 2013 Aceptado: 9 abril 2015 Corregido: 11 mayo 2015

\begin{abstract}
Resumen: Este artículo de divulgación presenta la descripción del programa educativo AULA.EDU, que tiene como propósito brindar oportunidades de estudio a estudiantes de educación media superior (bachillerato), en la modalidad de educación a distancia, a través de un convenio entre una institución universitaria (UANL) y una entidad gubernamental (Secretaría de Desarrollo Social). Se describen el contexto en que se desarrolla el programa, los propósitos, datos estadísticos, logros, lo no resuelto, las perspectivas futuras del programa y conclusiones.
\end{abstract}

Palabras clave: Educación media superior (bachillerato), educación a distancia, programa social, espacios alternativos no educativos, grupos vulnerables, educación y globalidad.

\begin{abstract}
This general interest article presents the description of the school program AULA.EDU, that has the main purpose of giving chance to study to middle and high level students in a distance learning course through an agreement between a college institution (UANL) and a government department (Social Development Department). It describes the context in which the program is developed, the purposes, the statistical data, achievements, impasses and perspectives of future programs and conclusions.
\end{abstract}

Key words: High school, distance learning, social program, non educative alternative spaces, vulnerable groups, education and global coverage.

En un mundo donde el conocimiento es el capital más significativo de las naciones, la educación se constituye en un pilar para el desarrollo; los sistemas educativos y los países que los impulsan están necesariamente obligados no tan solo a brindar educación, sino a ofrecer una educación de calidad; sin embargo, como lo menciona Coombs (1968) en el Reporte

1 Maestra en enseñanza de las ciencias en químicas, también maestra en enseñanza superior. Es profesora de tiempo completo y exdirectora de una escuela preparatoria en la UANL y actualmente es coordinadora de Proyectos Estratégicos en la Dirección de Estudios de Nivel Medio Superior de la misma universidad. Tiene seminarios de capacitación a nivel Internacional en Gestión Directiva, Centro Internacional de Estudios Pedagógicos CIEP en Francia; Gestión Directiva Academia Toulouse, Francia; Seminario Sociedad del Conocimiento Universidad Católica en Argentina, y CURSO IGLU México 2012. Tiene 22 ponencias en Congresos Internacionales de Investigación Educativa y en Congresos Nacionales de Formación Docente del Nivel Medio Superior ANUIES. Y los trabajos de investigación más recientes son La atención de salud en el adolescente de preparatorias de la UANL e Influencia de un programa de valores en los alumnos de la preparatoria 23, UANL. es_medina_t@hotmail.com 
Mundial de Educación La crisis mundial de la educación, a partir de la segunda mitad del siglo XX la demanda educativa supera por mucho la oferta que ofrecen los Estados, fenómeno no resuelto y que sigue presente en nuestros tiempos.

En el contexto de estos antecedentes y con el objetivo de alcanzar la cobertura educativa, se buscan alternativas basadas en la creatividad y la cooperación mutua para resolver el problema de la demanda, particularmente en países como México, en el que recientemente (2013), por disposición gubernamental, la educación media superior se ha declarado obligatoria y se integra, por tanto, al sistema nacional de educación básica.

De acuerdo con estas consideraciones y de otras que se mencionan a lo largo del documento, surge la propuesta de AULA.EDU, un proyecto destinado a ofrecer educación preparatoria a estudiantes del Estado de Nuevo León, México, particularmente a quienes pertenecen a grupos vulnerables que no pueden sufragarla, o bien, que por distintas razones no aprobaron el examen de ingreso.

El propósito del presente trabajo es divulgar las características del proyecto, los actores involucrados, el diagnóstico educacional, el análisis estadístico de los datos de demanda, el estudio del arte de la educación en ese nivel y las conclusiones que se derivan del desarrollo de ese programa socioeducativo. Así mismo se exponen algunas posturas sobre el futuro del programa, que en perspectiva puede constituirse en una propuesta educativa sólida, no tan solo para este país, sino también para otros países de América Latina.

El presente artículo de divulgación se construye a través del seguimiento de una estructura que permite abordar, en su primera parte, el problema desde lo global hasta lo local, citado en su contexto, y exponer la problemática del nivel educativo mencionado, con el fin de justificar la creación del proyecto AULA EDU. En el punto siguiente se aborda la descripción del proyecto de manera puntual y suficiente, desde su propósito hasta sus características, así mismo se incluye la caracterización de las instituciones involucradas y la condición presente de la problemática que se aborda.

Finalmente, en las conclusiones se destacan los logros y lo que no se ha resuelto, así como las recomendaciones que se consideran necesarias para el desarrollo futuro del programa.

\section{Preámbulo. Un acercamiento al problema}

Partiendo del hecho de que la educación tiene como misión desarrollar los talentos de las personas y todas sus capacidades de creación, lo que implica que cada quien pueda responsabilizarse de sí, realizar su proyecto personal y contribuir al desarrollo de la sociedad, se establece que la educación es un bien colectivo al que todos los grupos ciudadanos deben acceder; por tanto, en las decisiones que los países adoptan sobre educación, siempre predomina el principio de igualdad de oportunidades. Al respecto Delors (1996, p. 28) menciona: "Hay que ofrecer a niños y jóvenes lugares de aprendizaje, darles instrumentos para pensar y preparar su porvenir, y asegurar que sus perspectivas de futuro no se cierren".

El asunto de la equidad educativa y la igualdad de oportunidades no es nuevo, en el reporte mundial de educación "Aprender a ser", un diagnóstico educativo publicado por la UNESCO, Faure et al. (1973, p.144) afirman: 
Se debe aspirar a la igualdad de acceso a la educación con igualdad de oportunidades, un acceso amplio a los sistemas educativos con democracia de la educación; debido a que en el presente hay una presión sin precedentes, de la demanda de educación a todos los niveles y todas sus formas debido a una serie de necesidades e intereses de la sociedad actual.

Se parte del hecho que la educación, de una u otra forma, contribuye a determinar el destino de las sociedades en todas las fases de la evolución humana, y nunca ha cesado su desarrollo (Coombs, 1968). Estas son, esencialmente, las razones por las que el tema de la cobertura educativa merece la atención de las instituciones en México, además de atender una de las recomendaciones de la Organización para la Cooperación y Desarrollo Económicos (OCDE) para este país en el año de 1996, en el sentido de hacer realidad la equidad educativa, que implica ofrecer educación media superior a todas las personas candidatas que tengan la capacidad de recibirla; situación similar que afrontan los países latinoamericanos en los que el desafío es fortalecer la cobertura con equidad, especialmente en la población más desprotegida.

Sin embargo, el fenómeno es complejo y está ligado a la renovación en los procesos internos y a las formas de organización de los sistemas educativos para dar cobertura sin comprometer la calidad de la educación que se imparte. En relación con esta meta, lo observable es que, de acuerdo con las tendencias internacionales, los países de América Latina han extendido la educación obligatoria para abarcar, al menos, el ciclo de secundaria, pero algunos otros países, como el caso de México, han hecho obligatoria la educación básica hasta el ciclo de educación media superior (bachillerato) en todas sus modalidades (UNESCO, 2012).

Así, el sistema educativo mexicano y las instituciones que lo integran enfrentan diversas demandas sociales que exigen ser atendidas con la mayor urgencia y efectividad posibles, con el propósito de garantizar la participación activa y relevante de las escuelas y universidades en los proyectos de desarrollo social y económico del país, sin soslayar que tales demandas son también consecuencia del entorno particular de la educación media superior y superior, formación que cambia aceleradamente como resultado de los procesos de globalización, del desarrollo de la sociedad del conocimiento, de la innovación científico- tecnológica, de las rápidas transformaciones en la estructura del mercado laboral y ocupacional, de los procesos de transparencia y rendición de cuentas a la sociedad, y de una mayor exigencia por la pertinencia y calidad de la educación que imparten las instituciones.

Ante ello, la intervención de México en la aldea global tiene una estrecha relación con una educación media superior en expansión, debiendo preparar un mayor número de jóvenes a fin de concederles las condiciones que el marco internacional exige, ya que México enfrenta cada vez, con mayor frecuencia, la competencia de otros países en vías de desarrollo, que si bien no cuentan con una población calificada, o con una escolaridad más elevada, poseen mano de obra ideal para la producción de manufacturas simples que exigen escasas habilidades y que, por tanto, atraen la inversión de economías poderosas interesadas en abaratar los costos de inversión a través de mano de obra barata y bajos salarios, lo que afecta el potencial de México para incrementar sus ingresos.

Se debe considerar, además, que, en correspondencia con las tendencias demográficas y educativas que privan en México, el crecimiento más trascendente del sistema educativo nacional 
se localiza en el presente y en el futuro próximo inmediato de la población concurrente a los estudios del nivel medio superior, tanto por el número de jóvenes que cursa esta modalidad, como por quienes se encuentran en edad de cursarlo (Secretaría de Educación Pública, 2008; 2009).

Ante la circunstancias generadas por esta realidad demográfica educativa, la Universidad Autónoma de Nuevo León (UANL) busca responder con oportunidad y niveles reconocibles de calidad a las demandas sociales derivadas de los fenómenos mencionados con anterioridad, ello implica una gran capacidad organizativa por parte de la institución universitaria para: planear su desarrollo; formar a las nuevas generaciones de profesionales que atenderán esta demanda y crear e implementar los proyectos necesarios para orientar coherentemente y de manera articulada su quehacer ante la comunidad, en el logro de objetivos comunes, relevantes y ampliamente consensuados.

La Universidad Autónoma de Nuevo León (UANL) fue fundada en 1933 y es la tercera universidad más grande de la República Mexicana, constituyéndose en la institución pública más importante y con mayor oferta académica del noreste del país. En el ciclo escolar enero - junio 2014, la UANL atiende a una población escolar de 164.809 estudiantes en los niveles medio superior (bachillerato), superior y posgrado, tanto en los campus universitarios como en diversos planteles ubicados en 36 municipios del Estado de Nuevo León, ofertando un total de 256 programas educativos en los tres niveles anteriormente referidos, de los cuales 37 corresponden a bachillerato en sus diversas modalidades. La oferta educativa del nivel medio superior (bachillerato) contempla los de Bachillerato General, Bachillerato General a Distancia ${ }^{2}$, Bachillerato Bilingüe, Bachillerato Internacional, Bachillerato Bilingüe Progresivo en Inglés, Bachillerato Bilingüe Progresivo en Francés y Bachillerato Técnico, este último con un total de 31 programas. El número de escuelas preparatorias pertenecientes a la UANL en el Estado de Nuevo León asciende a 29 con un total de 38 planteles educativos (UANL, Plan de Desarrollo Institucional 2011).

En el plano organizacional, la Dirección de Estudios de Nivel Medio Superior (DENMS), coordinada por la Secretaría Académica de la Universidad Autónoma de Nuevo León, es el organismo con funciones asignadas que permiten dar respuesta al complejo y cambiante contexto que este nivel educativo implica; en particular, las que sustentan la operación de los programas académicos, dentro de un modelo educativo pertinente que fomente la equidad, la formación integral de estudiantes, el desarrollo y capacidades generales y específicas, lo cual permite a sus egresados y egresadas incorporarse a los estudios de licenciatura o bien al mundo laboral, tanto nacional como internacional.

No obstante lo anterior, tanto las autoridades universitarias como integrantes de la Dirección de Estudios de Nivel Medio Superior (DENMS) reconocen que uno de los principales retos que enfrenta la educación media superior (bachillerato), no solo en el Estado de Nuevo

2 En las escuelas preparatorias de la UANL que imparten estudios en su modalidad a distancia el alumnado realiza sus clases a través de medios tecnológicos combinados con sesiones presenciales y asistidas por una persona facilitadora o tutora. Los exámenes necesariamente en sesiones presenciales. Es una modalidad de estudio flexible que considera las limitaciones de tiempo y lugar que puede tener sus estudiantes. Esta modalidad se basa en un trabajo más independiente del alumnado, pero en contacto permanente con personal asesor y en interacción con sus compañeros y compañeras, con una participación estudiantil activa que recurre a los medios tecnológicos más utilizados como discos compactos, videocasetes, audio-casetes e internet. 
León sino en todo México, es fortalecer la cobertura y equidad, asegurando la igualdad de oportunidades educativas para toda la población mexicana (SEP, 2007; SENL, 2013).

En este sentido, se observa con preocupación que una cantidad importante de jóvenes y personas adultas no pueden realizar sus estudios en escuelas preparatorias del nivel medio superior de la UANL. En términos generales, el fenómeno se explica por tres razones fundamentales: en primer lugar por la falta de cupo en los diferentes planteles educativos; en segundo, por los resultados que obtienen los estudiantes examen de admisión denominado Proceso de asignación de espacios de las instituciones de educación media superior pública en el Estado y, en tercer lugar, por la falta de capacidad económica del estudiantado, lo que lo imposibilita para subragar sus colegiaturas. Resulta obvio decir que, para resolver la problemática de la equidad educativa, es urgente diseñar y desarrollar programas alternativos e innovadores que generen respuestas viables y pertinentes.

\section{Una propuesta concreta. Desarrollo del programa}

Ante esta situación, la Rectoría de la Universidad Autónoma de Nuevo León, a través de la Dirección de Estudios de Nivel Medio Superior (DENMS), gestionó, ante la Secretaría de Desarrollo Social del Estado de Nuevo León (SEDESOL), la creación de un programa interinstitucional que ofrece una alternativa para estudiar el bachillerato general a distancia a aspirantes con interés que así lo solicitan y cumplen con los requisitos que señalan los reglamentos de ingreso y permanencia, vigentes en la UANL. El programa se concretó a través del convenio denominado AULA.EDU. Este ofrece la posibilidad, al estudiantado, de cursar el bachillerato en sus propias comunidades, específicamente en los llamados centros comunitarios, instancias de difusión cultural patrocinados por el Gobierno Estatal que, en coordinación con las preparatorias pertenecientes a la UANL, han logrado coordinar espacios de estudio a distancia en la que el alumnado recibe asesoría académica para alcanzar las metas del proyecto personal e institucional. Quizás el punto más relevante del programa es la protección que AULA.EDU brinda a los grupos vulnerables de la sociedad, ya que la juventud integrante del proyecto, mediante becas, se exime de pago, por lo que su estatus económico social deja de ser un impedimento para sus estudios de nivel medio superior.

El convenio establece que en un lapso no mayor a cinco años se contará con quince espacios educativos, que incluyen equipos de cómputo, conexión a internet, plataforma ${ }^{3}$ Nexus - UANL, material bibliográfico, pizarrón, pero sobre todo, un aspecto relevante para la continuidad del proyecto, la designación de personal asesor en el uso de las tecnologías y en asuntos académicos. Estas tutorías asignadas por cada área del conocimiento tienen el propósito de orientar la formación de estudiantes que, por su edad y grado de madurez (adolescencia), requieren mayor apoyo.

Los criterios básicos para promover esta alternativa educativa y establecer los centros extraescolares de formación universitaria fueron:

3 Plataforma web que desde agosto de 2006 complementa el proceso educativo en la Universidad Autónoma de Nuevo León 
a) El radio de localización de los espacios educativos extraescolares se situará dentro del área metropolitana de Monterrey con ubicación en zonas geográficas cuyas familias tengan escasos recursos económicos.

b) En casos específicos se atenderán comunidades donde están concentrados migrantes e indígenas de otros estados de la república mexicana.

c) Otra consideración importante es que no exista, cerca del centro comunitario de SEDESOL, alguna institución de educación media superior, especialmente de carácter público

d) Finalmente se acordó instalar los centros educativos universitarios en municipios de Nuevo León con poblaciones iguales o superiores a 25,000 habitantes, sin alternativa educativa de la UANL.

Participan en el convenio AULA.EDU, los departamentos jurídicos de las dos instituciones involucradas SEDESOL y la UANL; la Universidad Autónoma de Nuevo León está a cargo de la dirección de este proyecto. Al mismo tiempo, para fortalecer y complementar el programa, se signó otro acuerdo entre la UANL y las editoriales responsables de la publicación de los libros de texto, elaborados por docentes del nivel medio superior de esta Casa de Estudios, para donar paquetes de libros a estudiantes de esta modalidad educativa.

En la concreción de la primera etapa, se hizo una invitación a participar de este proyecto a la población de tres zonas del área metropolitana de Monterrey, Nuevo León (con una población superior a 3, 000,000 de habitantes); donde los niveles de marginación son altos y no existen otros centros educativos del nivel medio superior de carácter público. En respuesta a esta convocatoria se presentaron 337 aspirantes a ingresar a la modalidad, en el proceso se aplicaron los lineamientos usuales de ingreso e inscripción en la Universidad Autónoma de Nuevo León. Finalmente, 289 aspirantes se aceptaron, por lo que se realizó su trámite de inscripción y el otorgamiento de becas completa de estudios a quienes lo solicitaran.

En las etapas subsecuentes, fueron formalizados otros centros educativos distribuidos en diferentes puntos tanto del área conurbada de Monterrey, así como del resto del Estado de Nuevo León, donde no existe oferta educativa de la UANL o de alguna otra institución de nivel medio superior. Es de destacar que cada espacio AULA.EDU implementado cuenta mínimamente con los requerimientos establecidos en el convenio: módulos con su respectivo equipo de cómputo, servicio de internet, plataforma Nexus - UANL, pizarrón y en cada un acervo bibliográfico oficial utilizado por las escuelas preparatorias de la UANL. Se cuenta asimismo con una persona responsable de lo administrativo y otra de lo académico por cada preparatoria, así como asesorías académicas para cada asignatura, con el fin de darle seguimiento al avance académico de cada estudiante. La Secretaría de Desarrollo Social aporta el personal responsable administrativo (1) y el académico (1) para cada espacio alternativo ubicado en el centro comunitario.

Se realizan, también, dos reuniones periódicas por semestre escolar por parte de este personal responsable tanto de SEDESOL como de la UANL, sesiones en las que se monitorea el avance escolar estudiantil, y se efectúan análisis de avances y retrocesos 
para la retroalimentación del proceso y para combatir los altos niveles de deserción escolar, característicos de este nivel.

Actualmente AULA.EDU cuenta con catorce centros comunitarios donde se han instalado espacios físicos y se contempla uno más, este se encontrará ubicado en la zona más desprotegida de Monterrey en cuanto a su nivel socioeconómico y baja escolaridad. A la fecha, el programa ha permitido graduar a 197 estudiantes pertenecientes a los tres primeros centros comunitarios inaugurados, y cuenta con una población cercana a 3000 estudiantes, que se distribuyen en los catorce espacios alternativos que se ubican en once municipios del Estado de Nuevo León y se inscriben en el mismo número de escuelas preparatorias de la UANL. Las metas del programa establecen que en un futuro próximo se alcanzará una cifra de 5000 estudiantes inscritos, en un tiempo máximo de dos años.

El Programa AULA.EDU se presentó oficialmente en III Congreso del Instituto de Gestión y Liderazgo Universitaria (IGLU) de la Organización Interamericana Universitaria (OUI), realizado en el Campus de la Pontificia Universidad Católica, en Lima, Perú, el 29 y 30 de abril del 2013. La temática del congreso versó sobre: La Gestión de las Instituciones de Educación Superior y las Estrategias Nacionales de Desarrollo, y la ponencia referida fue: Bachillerato a distancia, una alternativa de acceso a los estudios del nivel medio superior para fortalecer la equidad y ampliar la cobertura, particularmente en la población vulnerable del Estado de Nuevo León.

Vale decir que para concretar la propuesta y su posterior desarrollo, hubo que resolver los numerosos inconvenientes presentados en su implementación, factores de orden político, económico y social, a los que se suman, por supuesto, los inherentes al proceso educativo que implica la participación de los principales actores: personal de dirección y de administración, docentes y estudiantes, tanto de las escuelas preparatorias participantes como las de los centros comunitarios asignados al proyecto.

Es de destacar que otro de los retos a vencer es el limitado dominio en el uso de las diversas herramientas de las tecnologías de la información y comunicación (TIC) por parte de las futuras poblaciones estudiantiles, a lo que suma la adecuación tecnológica de los espacios físicos que fueron adaptados para esta esta modalidad educativa.

También, y quizá aún más significativo, el desafío mayor se centra en el esfuerzo que hay que realizar para dar viabilidad y permanencia al programa AULA.EDU como una opción atractiva y diferente, ya que en el pasado próximo se desarrollaron otros programas semejantes que no tuvieron el éxito esperado. Entre ellos destacan dos acuerdos con instituciones privadas en convenio con la Secretaría de Desarrollo Social (SEDESOL), que ofrecía a sus aspirantes la posibilidad de pagar cuotas escolares mínimas y asesorías académicas en sus propias instalaciones, pero que ante la imposibilidad del alumnado para sufragar las aportaciones escolares, fracasaron. En el caso concreto de este programa, se espera que siga representando una alternativa educativa viable para las personas que ya han iniciado, y que esperan concluir en un futuro próximo sus estudios del nivel medio superior.

En el plano académico existen otros aspectos por superar. En los últimos años se observó con preocupación, tanto en la Universidad Autónoma de Nuevo León como en el resto de las instituciones educativas públicas del Estado que ofertan la educación media superior, que 
un gran número de aspirantes que participaron en el Concurso de Ingreso a la Educación Media Superior (aplicado desde 1998 hasta 2011), no logró acceder a alguna de las distintas modalidades de estudio de bachillerato ofrecidas por los diversos planteles educativos.

Ante este hecho, y por recomendación de la OCDE, cuyos países miembros ofrecen a su ciudadanía una escolaridad básica obligatoria de 15 años, se estableció que a partir del año 2012, ante el Régimen de Obligatoriedad de la Educación Media Superior en México, el proceso de selección se transformara para dar lugar al Proceso de Asignación de Espacios de las Instituciones de Educación Media Superior Pública en el Estado de Nuevo León; con el objetivo de asegurar que un mayor número de egresados y egresadas de secundaria cuenten con un lugar para la realización de sus estudios de tipo medio superior.

Lo anterior puede representar un contrasentido porque, por una parte se pretende garantizar la aptitud de estudiantes para cursar un determinado nivel educativo; pero, por otra, se concibe que la obligatoriedad debe ser una garantía de acceso a la educación media superior. No obstante esta contradicción, ha quedado determinado que la incorporación de los candidatos y candidatas a este nivel se realizará de manera gradual y creciente a partir del ciclo escolar 2012-2013, y hasta el ciclo escolar 2021-2022 (Secretaría de Educación Pública, 2008). El proceso se sustenta en los mismos criterios que fueron aplicados en el Concurso de Ingreso anteriormente aplicado en el Estado de Nuevo León, y se señala que el puntaje obtenido en el examen será el argumento que utilizará quien concurse para asegurar su derecho a la admisión en alguna escuela preparatoria de su preferencia (SENL, 2013); en el caso de quienes no alcancen el puntaje requerido, se trasferirán a otras instituciones educativas designadas por el Estado.

Con el propósito de ilustrar estadísticamente lo mencionado, se presenta la tabla 1, en la que se puede advertir que hay un número importante de concursantes registrados sin un espacio asignado para sus estudios de bachillerato. Igualmente, existe otro número importante de personas no registradas, estas buscan trabajo en fuentes de empleo informales, porque la mayoría no cuenta ni con la edad mínima requerida ni con los estudios solicitados por la iniciativa privada o de servicios. Finalmente, otro porcentaje de jóvenes no estudian ni trabajan y constituyen un importante grupo de la población del Estado de Nuevo León.

Se estima también, de acuerdo con datos brindados por parte de la Secretaría de Educación de Nuevo León (SENL), que un número aproximado de doce mil jóvenes se integran a escuelas privadas del nivel medio superior. En conclusión y de acuerdo con datos extraoficiales, ya que no hay datos emanados de alguna instancia institucional pública o privada, un promedio anual de diez mil jóvenes se quedan sin estudiar y un número no especificado se integra a trabajos informales.

El problema es complejo y de gran magnitud si se reflexiona sobre el impacto social de tales datos, sin considerar a grupos de jóvenes que año tras año se suman a estas cifras negativas, y el número indeterminado de población migrante que llega a este Estado mexicano, especialmente de diferentes comunidades indígenas del país y de otras poblaciones que, con la esperanza de acceder a mejores niveles de vida, se suman al sector vulnerable que no tiene acceso a los estudios de bachillerato. Por otra parte, es importante señalar que el fenómeno no solo tiene carácter local sino además nacional e, incluso, internacional, si consideramos la 
Tabla 1

Ingreso a nivel medio superior en instituciones de carácter público (2010-2013)

\begin{tabular}{|c|c|c|c|c|c|c|c|}
\hline \multicolumn{3}{|c|}{$\begin{array}{c}\text { Egresados } \\
\text { Nivel Secundaria }\end{array}$} & \multirow{2}{*}{$\begin{array}{c}\text { Registrados } \\
35070\end{array}$} & \multirow{2}{*}{$\begin{array}{c}\text { Examinados } \\
34031\end{array}$} & \multirow{2}{*}{$\begin{array}{l}\text { Cupo } \\
23845\end{array}$} & \multirow{2}{*}{$\begin{array}{c}\text { Asignados } \\
26302\end{array}$} & \multirow{2}{*}{$\begin{array}{c}\begin{array}{r}\text { \%Aceptado } \\
\text { del Total de } \\
\text { Egresados }\end{array} \\
37.39\end{array}$} \\
\hline \multirow{2}{*}{2010} & 70345 & UANL & & & & & \\
\hline & & Total & 49413 & 47179 & 46845 & 45902 & 65.25 \\
\hline \multirow{2}{*}{2011} & 70893 & UANL & 36520 & 34883 & 25685 & 27501 & 38.79 \\
\hline & & Total & 51211 & 47743 & 46656 & 45679 & 64.43 \\
\hline \multirow{2}{*}{2012} & 71489 & UANL & 37815 & 35903 & 29146 & 29998 & 41.96 \\
\hline & & Total & 54798 & 51875 & 50876 & 48905 & 68.40 \\
\hline \multirow{2}{*}{2013} & 75975 & UANL & 38431 & 37109 & 31012 & 32897 & 43.29 \\
\hline & & Total & 56112 & 53678 & 51243 & 50765 & 66.85 \\
\hline
\end{tabular}

Nota: Secretaría de Educación Pública de Nuevo León y Centro de Evaluaciones, UANL.

realidad de los países de América Latina; en este sentido se destacan, en la siguiente parte del texto, otros aspectos de suma importancia a nivel institucional, que permiten justificar el proyecto AULA.EDU y darle continuidad al programa.

Uno de los aspectos que fundamentan el programa refiere al beneficio de otorgar doce años de educación a estudiantes en Iberoamérica, lo cual es, sin duda, el objetivo más ambicioso del proyecto Garantizar el Acceso a la Educación Planteado en Metas Educativas 2021 para los países que conforman Iberoamérica. Al respecto, los estudios del tema aseguran que recibir entre once y doce años de educación formal es la mejor alternativa para: contar con posibilidades reales de salir de la pobreza o para no caer en ella; para disponer de competencias con la finalidad de integrarse al sector productivo en nuevos empleos a lo largo de la vida; o bien para acceder al mundo laboral con una mejor remuneración. Los impulsos de cada nación deben descansar no solo en la ampliación de la cobertura en sí misma, sino además establecer condiciones necesarias que garanticen a la niñez y a la juventud su ingreso y permanencia en el sistema educativo (OEI para la Educación, la Ciencia y la Cultura, 2010).

Congruente con estos estudios, en el caso de México, recientemente, mediante la reforma a los Artículos $3^{\circ}$ y $31^{\circ}$ de su Constitución Política, se estableció la obligatoriedad de los estudios 
del tipo medio superior en el país. El decreto publicado en el Diario Oficial de la Federación, el 10 de febrero de 2012, establece que todo individuo tiene derecho a recibir educación: Se asienta que la federación, los estados y municipios impartirán educación preescolar, primaria, secundaria y media superior. La educación preescolar, primaria y secundaria que conforman la educación básica y la media superior serán obligatorias. La reforma mencionada, en su artículo segundo transitorio, establece que de manera gradual, a partir del ciclo escolar 2012 -2013, y hasta el ciclo escolar 2021 - 2022, el Estado y sus organismos descentralizados deberán generar condiciones para hacer realidad la obligatoriedad de este tipo de estudios (Secretaría de Educación Pública, 2008).

En cuanto a la perspectiva de la demanda potencial de este servicio educativo en los próximos diez años en el Estado de Nuevo León, se parte del número de jóvenes que concluirán el nivel básico de educación, haciendo una estimación de las personas egresadas de secundaria (ver tabla 2). En tal proyección, se considera que el número puede variar en cada ciclo escolar, por motivo de cambios en la dinámica geográfica, cobertura de servicios y la eficiencia del sistema educativo durante el tránsito del estudiantado en este nivel de formación académica. La estimación de la demanda futura se hace desde tres escenarios distintos que se denominan: inercial, medio y acelerado, partiendo del ciclo escolar 2012 2013 hasta el 2021 - 2022.

Tabla 2

Comparación de los tres escenarios del cambio previsible en la capacidad de atención en la educación media superior en relación al cambio en el número de personas egresadas de educación secundaria

\begin{tabular}{|c|c|c|c|c|c|}
\hline \multirow[t]{2}{*}{ Ciclo Escolar } & \multicolumn{3}{|c|}{ Egresados } & \multicolumn{2}{|c|}{$\begin{array}{c}\text { Índice de crecimiento } \\
\text { inercial }=100\end{array}$} \\
\hline & Inercial & Medio & Acelerado & Medio & Acelerado \\
\hline $2012-2013$ & 75,817 & 75,954 & 76,461 & 100.2 & 100.8 \\
\hline $2013-2014$ & 74,316 & 74,683 & 75,486 & 100.5 & 101.6 \\
\hline $2014-2015$ & 80,632 & 81,445 & 82,771 & 101.0 & 102.7 \\
\hline $2015-2016$ & 87,566 & 88,691 & 90,752 & 101.3 & 103.6 \\
\hline $2016-2017$ & 94,305 & 95,774 & 98,801 & 101.6 & 104.8 \\
\hline $2017-2018$ & 79,744 & 81,044 & 84,560 & 101.6 & 106.0 \\
\hline $2018-2019$ & 78,414 & 79,892 & 84,261 & 101.9 & 107.5 \\
\hline $2019-2020$ & 78,124 & 80,935 & 85,165 & 103.6 & 109.0 \\
\hline $2020-2021$ & 78,904 & 84,552 & 87,356 & 107.2 & 110.7 \\
\hline $2021-2022$ & 79,331 & 89,288 & 89,288 & 112.6 & 112.6 \\
\hline
\end{tabular}

Nota: Secretaría de Educación Nuevo León. 
Atendiendo al procedimiento que consiste en evaluar el progreso de las cohortes de estudiantes por el sistema educativo, que comprende desde segundo año de preescolar hasta el término de los estudios de secundaria, y a que la eficiencia terminal continuará su tendencia histórica en el ciclo escolar 2012 - 2013 de 87.4\%, en el ciclo escolar 2016 - 2017, la eficiencia terminal será del 87.9\%, mientras que para el ciclo escolar 2021 - 2022 será del 88.8\%. Así, la población escolar que egresó en agosto de 2012 - 2013, que es de aproximadamente 70,619, un lustro después será de 94,305 , lo que constituye un 33.5\% adicional de crecimiento; sin embargo, a partir del año 2017 - 2018 se considera una reducción drástica en la que la población estudiantil no alcanzará un número mayor de 79,400 durante cada año escolar hasta el 2022.

Para el año 2017, de seguir la tendencia actual y tomando en cuenta el escenario inercial de personas egresadas de secundaria de acuerdo con la tabla 2, relacionando el número total de egresos de secundaria y el porcentaje aceptado por Universidad Autónoma de Nuevo León (UANL), el reto para esta institución educativa será el de ampliar su oferta educativa significativamente hasta alcanzar un número 39,570 estudiantes en su primer ingreso, lo que constituye un 25.6\% adicional con respecto a su índice de aceptación en el ciclo 2012-2013; aunque, por otra parte, para el año escolar 2017 - 2018 el número de personas aceptadas tenderá a la baja, la cual es muy semejante a la matrícula de los años 2011-2012 y 2012-2013. Se debe suponer, adicionalmente, que la obligatoriedad es paulatina y al hacerse efectiva, los datos aquí presentados pudieran cambiar radicalmente, con lo cual la situación a futuro sobre el ingreso a bachillerato se complicarían aún más.

A continuación, en seguida en la figura 1 se puede observar la tendencia de la población escolar que pretenderá su ingreso al bachillerato en sus diferentes modalidades de estudio que ofrece la Universidad Autónoma de Nuevo León.

Población Escolar a Primer Ingreso

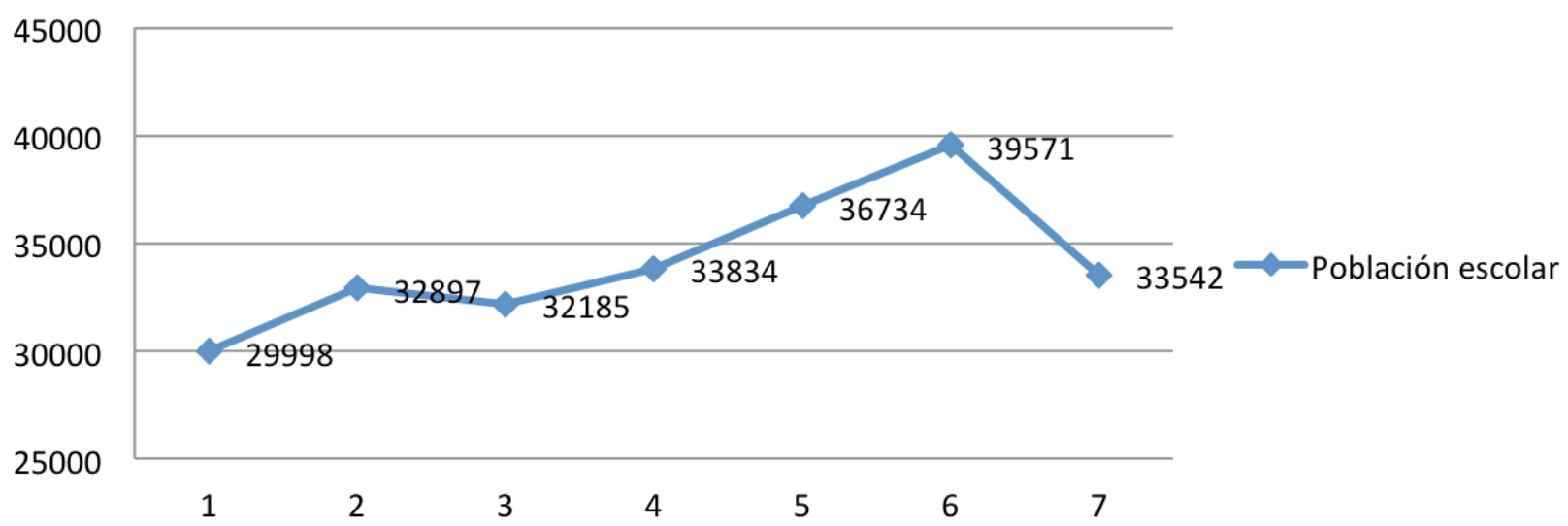

Nota: Dirección de Estudios de Nivel Medio Superior de la UANL.

Figura 1. Población escolar tendiente a ingresar en nivel medio superior en la UANL durante los próximos cinco años. 
Para atender esta situación, la Universidad Autónoma de Nuevo León (UANL), como parte de su responsabilidad social y sin soslayar la calidad de la educación media superior, con el propósito de promover el acceso a este nivel educativo, impulsa en la actualidad y de manera permanente una política de ampliación de su oferta educativa a través de diferentes modalidades: presencial y a distancia, entre la que se incluye AULA.EDU, descrita en este documento, y que posibilita la atención de las áreas marginales, estratégicas y prioritarias para el desarrollo de grupos poblacionales vulnerables.

Sin embargo, y no obstante que el programa está enfocado en atender la demanda de educativa de educación media superior (bachillerato) de los grupos vulnerables, esta Institución Universitaria, conocedora de que la educación media superior en México está actualmente en proceso de reforma y consolidación, acorde con las metas establecidas en el Plan Nacional de Desarrollo (PND) México 2007-2012, concibe las políticas de calidad educativa como una herramienta importante para elevar el desarrollo de la persona y, a su vez, promover el crecimiento económico. Se declara, por tanto, que la educación de calidad debe formar estudiantes con los niveles de destrezas, habilidades, conocimientos y técnicas que demanda el mercado de trabajo y se define en cinco rubros: cobertura, equidad, eficacia, eficiencia y pertinencia. Con ello, la Universidad Autónoma de Nuevo León pretende, particularmente en este nivel educativo, dar atención a los retos y problemáticas del mejoramiento de la calidad $y$, con esta propuesta en particular (AULA.EDU), aspirar a la cobertura y equidad educativa.

\section{Conclusiones}

AULA.EDU surgió como un proyecto innovador mediante el cual la Universidad Autónoma de Nuevo León y la Secretaría de Desarrollo Social del Estado establecen una sinergia de trabajo y asumen el compromiso social de ofrecer una nueva alternativa de estudios de bachillerato general a distancia, a estudiantes de sectores desprotegidos económicamente que conforman la parte más sensible de la sociedad. Además, conocedora de esta compleja situación, que forma parte ya de la exigencia social, afronta con decisión y da solución a problemas que pueden ser generadores de algunas complicaciones de índole social en el futuro próximo.

A la fecha son muy pocas las instituciones de carácter público o privado que han ofrecido un compromiso dentro del Estado de Nuevo León, con miras a convertirse en un proyecto factible y veraz para la población más vulnerable; sectores que se ven favorecidos al ofrecer a sus estudiantes en edad escolar la posibilidad de acceder a un nivel de estudios más alto y al mismo tiempo disminuir el porcentaje de jóvenes candidatos a continuar en su actual estatus social. Una referencia importante para valorar el éxito de AULA.EDU, parte de los siguientes datos: La inscripción inicial fue de 289 estudiantes, de esta se graduaron 197, y actualmente 48 del grupo inicial cursan estudios de nivel superior.

Actualmente, se siguen realizando esfuerzos conjuntos por mejorar el acompañamiento con personal tutor, además las escuelas preparatorias de la UANL que participan del proyecto decidieron ampliar el tiempo de asesorías académicas para el estudiantado de AULA.EDU y lo invita a participar activamente de sus eventos académicos, culturales y deportivos dentro de la Universidad Autónoma de Nuevo León. 
Con estas acciones se espera que las nuevas generaciones egresadas de AULA.EDU tengan un mejor rendimiento escolar que consolide los estándares de eficiencia terminal. El proyecto es tan ambicioso que se aspira a que en el futuro cercano la población de jóvenes formada en esta modalidad a distancia en un espacio extraescolar sea la misma cantidad que se gradúa en la modalidad presencial; mediante un programa que desarrolla su pleno potencial y les transforma en personas responsables, productivas y éticas, y que les posibilita el acceso a mejores niveles de vida, mediante su inserción positiva en la sociedad.

\section{Referencias}

Coombs, P. H. (1968). La crisis mundial de la educación. Nueva York. EUA: Oxford University Press.

Cámara de Diputados del H. Congreso de la Unión. (2012). Decreto por el que se declara reformado el párrafo primero; el inciso c) de la fracción II y la fracción $V$ del artículo 3o., y la fracción I del artículo 31 de la Constitución Política de los Estados Unidos Mexicanos (DOF 09-02-2012). Recuperado de www.diputados.gob.mx/LeyesBiblio/ proceso/lxi/204 DOF 09feb12.pdf

Delors, J. (1996). Compendio La educación encierra un tesoro. Informe a la UNESCO de la Comisión Internacional sobre la Educación para el Siglo XXI. Recuperado de http:/l unesdoc.unesco.org/images/0010/001095/109590so.pdf

Delors, J. (1996). La educación encierra un tesoro. Informe a la UNESCO de la Comisión Internacional sobre la educación para el siglo XXI. México: Santillana.

Faure E., Herrera, F., Kaddoura, A-R., Lopes, H., Petrovski, A., Rahnema, M. y Champion, F. (1973). Aprender a ser. La educación del futuro. España: Alianza UNESCO.

Gobierno de los Estados Unidos Mexicanos (2007). Plan Nacional de Desarrollo 2007-2012. Recuperado de www.cenidet.edu.mx/docs/pnd 2007 2012.pdf

Nl.gob.mx. (2013). Aplican a mil 400 personas examen de admisión para ingresar a bachillerato a distancia a través de la Red de Centros Comunitarios. Recuperado de http://www.nl.gob.mx/noticias/aplican-mil-400-personas-examen-de-admision-paraingresar-bachillerato-distancia-traves-de

Organización de Estados Iberoamericanos para la Educación, la Ciencia y la Cultura (OEI). (2010). Metas educativas 2021. Recuperado de www.oei.es/metas2021/libro.htm

Secretaría de Educación de Nuevo León. (2013). Fortalecimiento integral de la educación media superior del Estado de Nuevo León. PROFIDEMS. México: Autor. 
Secretaría de Educación Pública. (2007). Reforma integral de la educación media superior. La creación de un sistema nacional de bachillerato en un marco de diversidad. Recuperado de http://www. ofmx.com.mx/documentos/pdf/RIEMS Creacion Sistema Nacional de Bachillerato.pdf

Secretaría de Educación Pública. (2008). Acuerdo número 442 por el que se establece el Sistema Nacional de Bachillerato en un marco de diversidad. Recuperado de www.sems.gob.mx/ work/models/sems/Resource/11435/1/images/5 1 acuerdo numero 442 establece snb.pdf

Secretaría de Educación Pública. (2009). Acuerdo 488 por el que se modifican los diversos números 442, 444 y 447 por los que se establecen: el Sistema Nacional de Bachillerato en un marco de diversidad; las competencias que constituyen el marco curricular común del Sistema Nacional de Bachillerato, así como las competencias docentes para quienes impartan educación media superior en la modalidad escolarizada, respectivamente. Recuperado de www.sep.gob.mx/work/models/sep1/Resource/7aa2c3ff-aab8-479f-ad93db49d0a1108a/a488.pdf

UNESCO. (2012). Situación educativa de América Latina y el Caribe. Hacia una educación para todos 2015. Recuperado de www.orealc.cl/educacionpost2015/wp...dir/.../ efainformefinaldef57.pdf

Universidad Autónoma de Nuevo León. (2014). Egresa primera generación de preparatoria aula.edu. Recuperado de http://www.uanl.mx/noticias/academico/egresa-primerageneracion-de-preparatoria-aulaedu.html

Universidad Autónoma de Nuevo León. (2008). Bachillerato general a distancia. Nivel medio superior. Recuperado de www.uanl.mx/oferta/bachillerato-general-distancia.html

Universidad Autónoma de Nuevo León. (2005). Leyes y Reglamentos de la Universidad Autónoma de Nuevo León. Ley Orgánica. Honorable Consejo Universitario. Secretaría General. México: Autor.

Universidad Autónoma de Nuevo León. (2011). Manual organizacional. Recuperado de www. uanl.mx/sites/default/files/.../manual-de-organizacion-2014.pdf

Universidad Autónoma de Nuevo León. (2008). Plan de Desarrollo del Sistema de Educación Media Superior. Recuperado de www.uanl.mx/sites/default/files/PDI NMS11-junio2011.pdf

Universidad Autónoma de Nuevo León. (2006). Plan de Desarrollo Institucional UANL 20072012. Recuperado de www.uanl.mx/sites/default/.../plan-desarrollo-2007-2012-v09.pdf

Universidad Autónoma de Nuevo León. (2011). Plan de Desarrollo Institucional UANL 2012 - 2020. Recuperado de www.uanl.mx/sites/default/files/.../pdi-2020-26abril.pdf 